\title{
Neutrophil accumulation and NET release contribute to thrombosis in HIT
}

\author{
Kandace Gollomp, ${ }^{1,2}$ Minna Kim, ${ }^{1}$ Ian Johnston, ${ }^{3}$ Vincent Hayes, ${ }^{4}$ John Welsh, ${ }^{5}$ \\ Gowthami M. Arepally, ${ }^{6}$ Mark Kahn, ${ }^{5}$ Michele P. Lambert, ${ }^{1,2}$ Adam Cuker, ${ }^{4,5}$ Douglas B. Cines, ${ }^{4,5}$ \\ Lubica Rauova, ${ }^{1,2}$ M. Anna Kowalska, ${ }^{1,7}$ and Mortimer Poncz, \\ 'Division of Hematology, Children's Hospital of Philadelphia, Philadelphia, Pennsylvania, USA. ²Department of Pediatrics, \\ ${ }^{3}$ Department of Pharmacology, ${ }^{4}$ Department of Pathology, and ${ }^{5}$ Department of Medicine, Perelman School of Medicine, \\ University of Pennsylvania, Philadelphia, Pennsylvania, USA. ${ }^{6}$ Deparment of Medicine, Duke University School of \\ Medicine, Durham, North Carolina, USA. ${ }^{7}$ Institute of Medical Biology, Polish Academy of Science, Lodz, Poland.
}

\begin{abstract}
Heparin-induced thrombocytopenia (HIT) is an immune-mediated thrombocytopenic disorder associated with a severe prothrombotic state. We investigated whether neutrophils and neutrophil extracellular traps (NETs) contribute to the development of thrombosis in HIT. Using an endothelialized microfluidic system and a murine passive immunization model, we show that HIT induction leads to increased neutrophil adherence to venous endothelium. In HIT mice, endothelial adherence is enhanced immediately downstream of nascent venous thrombi, after which neutrophils undergo retrograde migration via a CXCR2-dependent mechanism to accumulate into the thrombi. Using a microfluidic system, we found that PF4 binds to NETs, leading them to become compact and DNase resistant. PF4-NET complexes selectively bind HIT antibodies, which further protect them from nuclease digestion. In HIT mice, inhibition of NET formation through Padi4 gene disruption or DNase treatment limited venous thrombus size. PAD4 inactivation did affect arterial thrombi or severity of thrombocytopenia in HIT. Thus, neutrophil activation contributes to the development of venous thrombosis in HIT by enhancing neutrophil-endothelial adhesion and neutrophil clot infiltration, where incorporated PF4-NET-HIT antibody complexes lead to thrombosis propagation. Inhibition of neutrophil endothelial adhesion, prevention of neutrophil chemokine-dependent recruitment of neutrophils to thrombi, or suppression of NET release should be explored as strategies to prevent venous thrombosis in HIT.
\end{abstract}

Conflict of interest: The authors have declared that no conflict of interest exists.

Submitted: December 26, 2017 Accepted: August 14, 2018 Published: September 20, 2018

Reference information: JCI Insight. 2018;3(18):e99445. https://doi.org/10.1172/jci. insight. 99445.

\section{Introduction}

Heparin-induced thrombocytopenia (HIT) is an immune-mediated thrombocytopenic disorder, characterized by venous and arterial thrombi (1). Even with early recognition and initiation of appropriate therapy, thromboembolic complications still occur (2). The current standard of care for patients with suspected HIT involves intense anticoagulation that carries the risk of significant bleeding and only provides partial protection against recurrent thromboembolic events (3). While avoidance of heparin exposure may prevent the development of HIT (4), there are clinical settings where heparin remains irreplaceable (5). An improved understanding of the pathobiology of HIT may help identify targeted therapies to prevent thrombosis without subjecting patients to the risk of intense anticoagulation. These insights may also be beneficial in the treatment of other inflammatory, prothrombotic conditions, ranging from antiphospholipid syndrome (6) to sepsis (7).

HIT is caused by the development of pathogenic antibodies (HIT antibodies) that recognize complexes of heparin and the platelet-specific human chemokine platelet factor 4 (PF4 also known as CXCL4) (8), which is released in high concentrations at sites of platelet activation and then binds to surface glycosaminoglycans (GAGs) on platelets (9), monocytes (10), endothelial cells (11), and neutrophils (12). HIT antibodies cross-react with PF4-GAG complexes, after which their Fc termini engage Fc $\gamma$ RIIa receptors, leading to cell activation (13). Although the high risk of thrombosis observed in HIT has classically been attributed to the assembly of PF4-GAG complexes on platelets, these complexes also form on the surface of other cells types, including monocytes (10) and neutrophils (14). Moreover, we recently showed that PF4 and HIT-like antibodies bind predominantly to the endothelial surface after vessel wall injury, leading to an increase in the extent of endothelial damage (11). 
There are multiple factors that suggest that neutrophils may contribute to the pathogenesis of HIT. Human neutrophils express Fc $\gamma$ RIIa (15), and neutrophil activation via this receptor promotes increased phagocytosis, degranulation, and generation of reactive oxygen species (16). PF4 and HIT antibodies have been shown to induce integrin expression on neutrophils (14) and enhance the formation of neutrophil-platelet aggregates (17). Myeloperoxidase (MPO) levels have also been found to be elevated in plasma obtained from patients with HIT compared with plasma from patients with other causes of thrombocytopenia (17), suggesting that HIT is associated with neutrophil activation. In the setting of infection or inflammation, activated neutrophils can contribute to thrombus formation through neutrophil extracellular traps (NETs), release chromatin that can trap platelets, bind clotting factors, and deactivate natural anticoagulants (18).

These findings led us to hypothesize that neutrophils activated by HIT antibodies may contribute to the prothrombotic nature of HIT. To address this question, we performed studies using a microfluidic system (11) and a passive immunization murine model of HIT (19). In both systems, we found that exposure to HIT antibodies lead to enhanced neutrophil adhesion to the venous endothelium. Following cremaster venule laser injury in the HIT murine model, we observed that neutrophils adhered to the downstream endothelium and then followed a chemogradient that induced a retrograde migration into the growing thrombi. When activated neutrophils undergo NETosis during HIT, their released NETs were bound by PF4, leading to their physical compaction. These PF4-NET complexes bound HIT antibodies and became resistant to circulating DNases. Additional passive murine immunization HIT studies in mice that are resistant to NET formation due to a deficiency in the gene that encodes peptidyl arginine deiminase 4 (PAD4), an enzyme that induces chromatin decondensation through the citrullination of histone lysine residues (20), confirmed that NETosis plays a role in the murine model of HIT. Corollary analysis of clinical samples obtained from patients diagnosed with HIT suggested that NET formation influences the disease in humans. The relevance of these studies to the development of the prothrombotic state in HIT and potential new targets for therapy are discussed.

\section{Results}

Enhanced neutrophil adhesion to the venular endothelium in HIT. Neutrophils are activated when exposed to PF4 and HIT antibodies (14). To determine if this activation might effect neutrophil-endothelial cell interactions, we infused calcein-AM-labeled whole blood through microfluidic channels lined with human umbilical vein endothelial cells (HUVECs) and quantified cell adhesion over time. We observed scant leukocyte-endothelium adhesion in unstimulated channels that was unchanged by the addition of the HIT-like monoclonal antibody KKO or the isotype control TRA (data not shown). However, when the HUVECs were pretreated with TNF- $\alpha$, there was a 2-fold increase in the number of adherent leukocytes in samples incubated with $\mathrm{KKO}$, which induces the release of endogenous PF4 from platelets (19), compared with samples treated with the isotype control TRA (Figure 1, A-C, and Supplemental Video 1; supplemental material available online with this article; https://doi.org/10.1172/jci.insight.99445DS1). We did not observe that TNF- $\alpha$ treatment led to an increase in KKO binding to the HUVECs (Supplemental Figure 2), supporting that the increase in leukocyte adhesion seen in vivo was at least in part a result of leukocyte rather than endothelial activation. We then asked if these findings could be recapitulated in vivo using a murine HIT model following injection of $\mathrm{KKO}(21)$. Within 30 minutes of $\mathrm{KKO}$ infusion, we observed increased neutrophil rolling and adhesion to uninjured cremaster muscle venules, but no neutrophil rolling or adhesion occurred in uninjured cremaster arterioles before or after the animals were exposed to KKO (Figure 1, D and E, and Supplemental Video 2). We similarly observed an increase in neutrophil adhesion in the femoral vein 10 minutes following treatment with KKO (Figure 1, F and G, and Supplemental Video 3).

Enhanced neutrophil involvement in venular thrombosis in HIT. We next asked whether exposure to HIT antibodies influences neutrophil incorporation into thrombi in vivo in the murine model of HIT. Cremaster vessels were injured at time 0 , and adherent neutrophils and platelets were quantified at 5 minutes. The mice were then infused with KKO or TRA, and the thrombi were reexamined at 60 minutes. While there was an increase in platelet accumulation in arterioles following HIT induction, only a small number of neutrophils were incorporated into arteriolar thrombi, with a small but significant increase following treatment with KKO (Figure 2). At sites of venular injury prior to HIT induction, there was platelet and fibrin accumulation (Figure 2B and Supplemental Figure 3, respectively), but only a small number of neutrophils adhered to these thrombi (Figure 2, A and C). In contrast, following HIT induction, there was a marked increase in neutrophil accumulation within venular thrombi (Figure 2C), with a minimal change in fibrin 
A
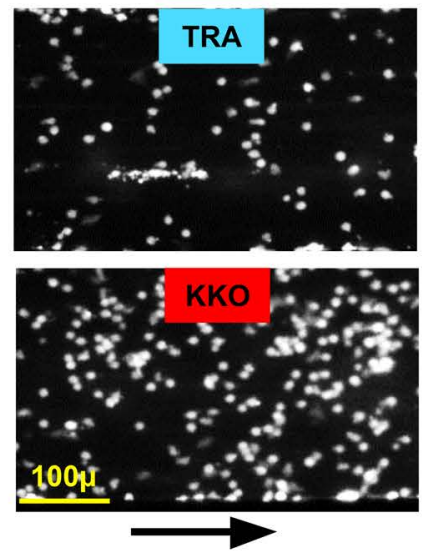

B

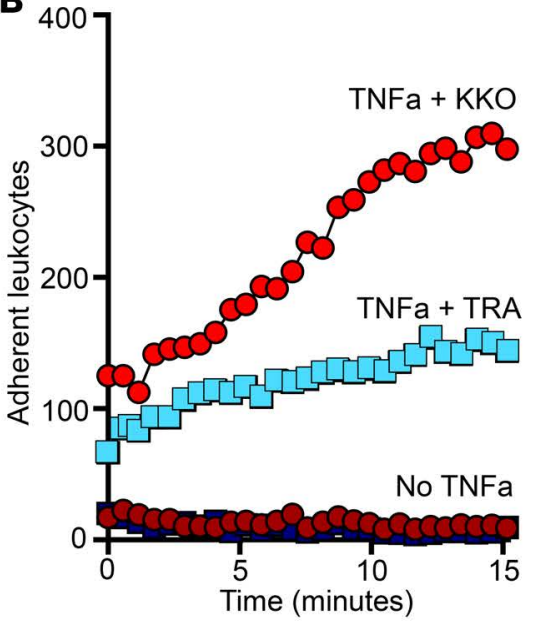

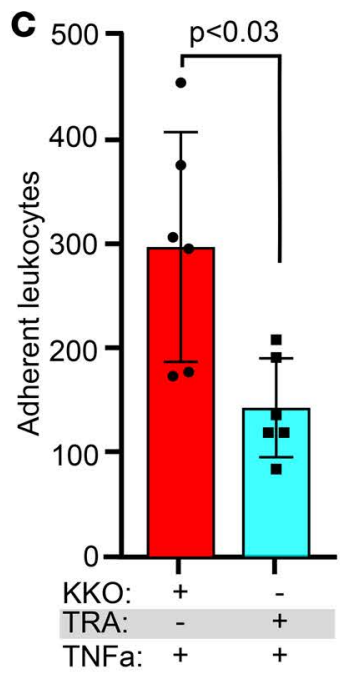

D

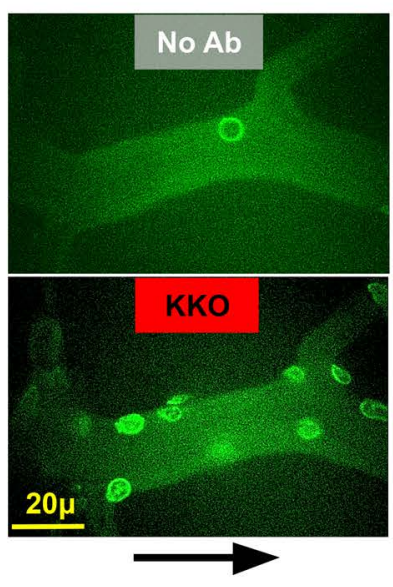

E

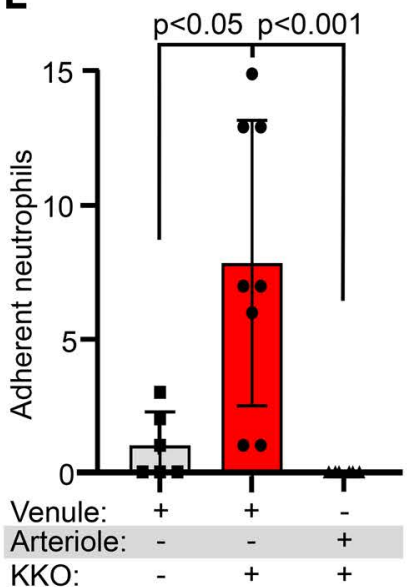

$\mathbf{F}$

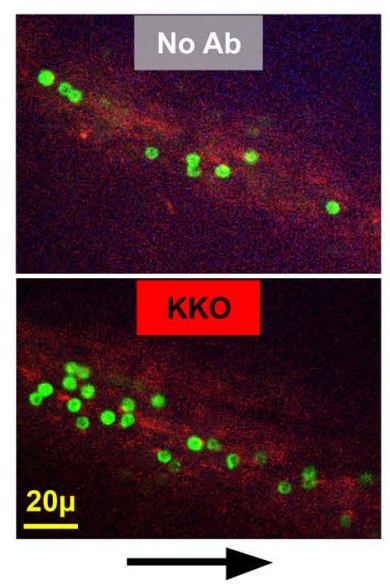

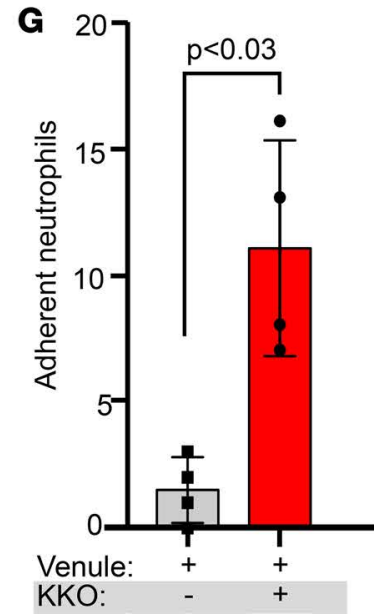

Figure 1. Enhanced leukocyte-endothelial adhesion in HIT. (A) Calcein-AM-labeled blood incubated with KKO (red) or TRA (blue) was infused through HUVEC-lined channels exposed to TNF- $\alpha$. Representative wide-field image of leukocytes (white) adhering to the endothelium is shown. An arrow indicating direction of flow is included. Scale bar: $100 \mu$. Image was obtained with an Axio Observer Z1 inverted microscope (original magnification $\times 10)$. (B) Leukocyte-endothelial adhesion was quantified for KKO (red) and TRA (blue). Six channels were studied in each TNF- $\alpha$-exposed arm (lighter colors) and three to five channels were studied in the unexposed arms (darker colors). (C) The final number of adherent leukocytes at 15 minutes is shown as mean \pm 1 SD. $n=$ 6 per arm. Comparative analysis was performed by Student's $t$ test. (D) Representative confocal image of neutrophils rolling in a venule before and after KKO infusion. Neutrophils were stained using anti-Ly-6G F(ab') fragment (green). An arrow indicating direction of flow is included. Scale bar: $20 \mu$. Images were obtained with an Olympus BX61WI microscope with a $\times 40 / 0.8$ numeric aperture water-immersion objective lens. (E) Neutrophil adhesion to cremaster arterioles and venules was studied in the HIT murine model prior to and 30 minutes after exposure to KKO. Adhesion was defined as neutrophil immobilization for $\geq 30$ seconds. 6 veins were studied without KKO exposure, 8 veins were studied after KKO injection, and 6 arterioles were studied after KKO injection. Statistical comparison of binding was preformed using a Kruskal-Wallis 1-sided ANOVA. (F) Representative confocal image of neutrophil rolling and adhering to the femoral vein before and 15 minutes after the infusion of KKO. Images are as in D. An arrow indicating direction of flow is included. Scale bar: $20 \mu$. (G) Neutrophil adhesion to the femoral vein with or without KKO infusion as in F. $n=4$ per arm. Statistical comparison was performed by Student's $t$ test.

accumulation (Supplemental Figure 3) and platelet volume (Figure 2B). The rise in neutrophil accumulation was not observed following TRA infusion (Figure 2).

A CXCR2-dependent retrograde migration of neutrophils into venular thrombi in HIT. To better understand the process of neutrophil accumulation in venular thrombi in HIT, we induced laser injuries in venules following KKO infusion and monitored platelet and neutrophil accumulation in these lesions over 5 minutes. While platelets quickly adhered directly to the site of venous injury, most neutrophils initially bound to the endothelium 30-40 microns downstream of the injury (Figure 3A and Supplemental Videos 4 and 5). This finding is likely due to a combination of increased neutrophil adhesiveness induced by KKO exposure, documented in Figure 1, in addition to activation of the endothelium downstream of cremaster laser injuries in HIT that we have previously described (11). These neutrophils subsequently migrated in a retrograde direction into the evolving thrombus (Figure 3A and Supplemental Videos 4 and 5) and 60 minutes following the 

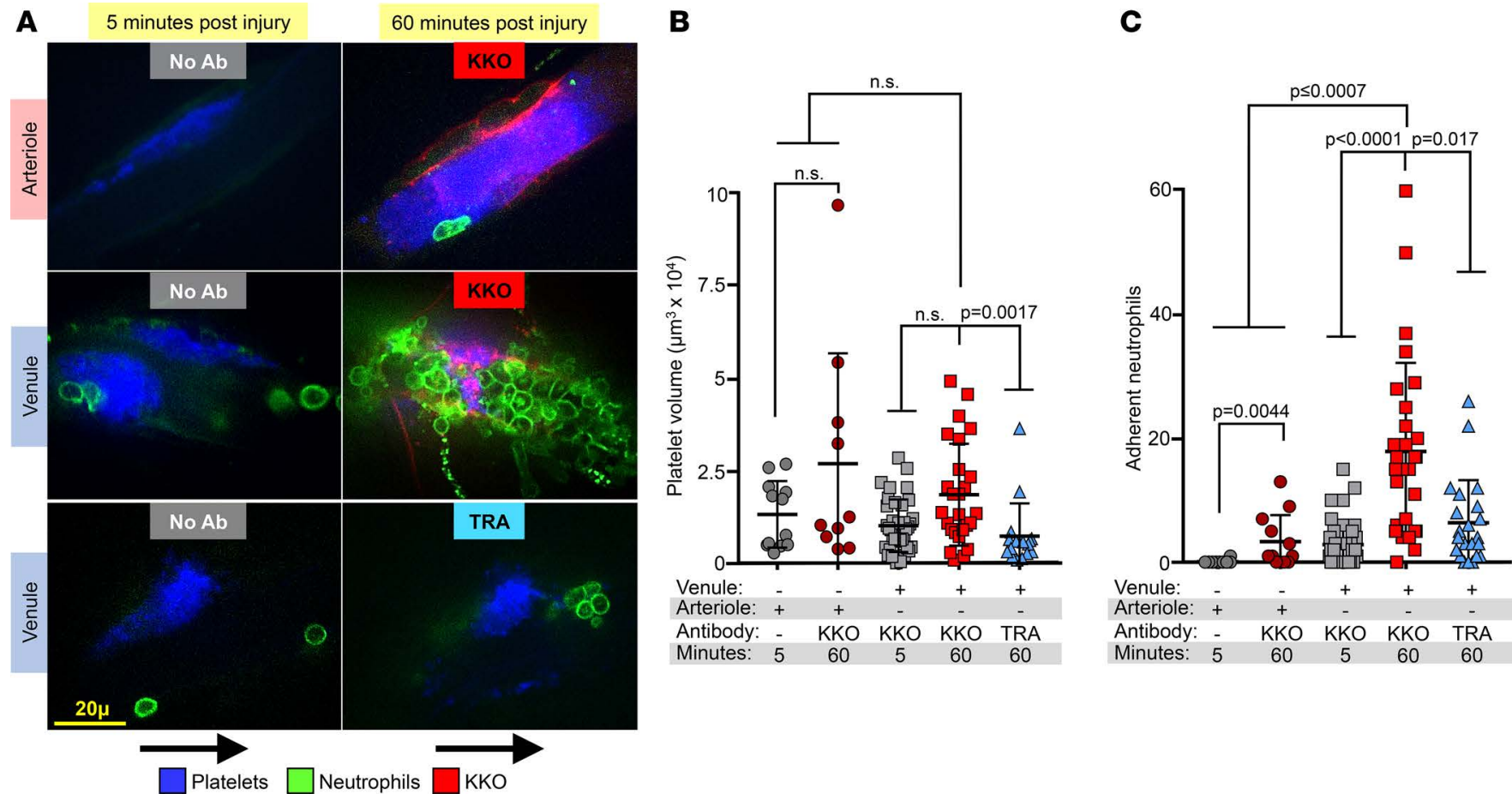

Figure 2. Effect of HIT of neutrophil accumulation in cremaster vessels before and after injury. (A) Representative confocal images from cremaster arteriole and venule laser injuries showing platelets labeled with anti-CD41 (dark blue) and neutrophils labeled with anti-Ly-6C (green). Paired images from the same vessel taken at 5 and 60 minutes following laser injury. KKO or TRA were infused intravenously beginning at minute 5 after injury. An arrow indicating direction of flow is included. Scale bar: $20 \mu$. Same microscope and acquisition software as in Figure 1D. (B and C) Quantification of adherent neutrophils and platelets in the same thrombi. Twenty-eight injuries were made in twelve KKO-treated mice. Sixteen injuries were made in four TRA-treated mice. Twelve arteriole injuries were made in three untreated and three KKO-treated mice. Individual data points and mean \pm 1 SD are shown. Comparative statistical analysis between 3 or more groups was performed by Kruskal-Wallis 1-way ANOVA and comparisons between 2 groups was performed with a Student's $t$ test.

initial injury, a significantly higher number of neutrophils was present in venular thrombi in mice exposed to KKO compared with those infused with the isotype control TRA (Figure 3 and Supplemental Video 6). We hypothesized that retrograde migration of adherent neutrophils into the thrombi was induced by a chemoattractant released by activated platelets in the clots. Activated platelets release several chemokines from their $\alpha$ granules that cause neutrophil migration by engaging neutrophil surface CXCR2 (22). To test whether the retrograde recruitment of neutrophils to venular thrombi in HIT was chemokine dependent, we treated the mice with the CXCR2 antagonist SCH527123 (22) prior to the induction of HIT. We observed that thrombi in mice treated with SCH527123 before KKO infusion contained significantly fewer neutrophils within their thrombi (Figure 3). Furthermore, these neutrophils were located at the periphery of the platelet-rich thrombi and not embedded within them.

In vitro studies of PF4-NET interactions. After observing marked HIT-induced neutrophil infiltration in venular thrombi, we hypothesized that adherent neutrophils contribute to venous thrombi in HIT, in part, through the release of NETs, as described in deep venous thrombosis (23). Moreover, platelet activation, one of the hallmarks of HIT, can initiate NETosis in inflammatory states $(24,25)$. Activated platelets release high levels of PF4, which forms high-molecular weight aggregates with various polyanions, producing antigens that are recognized by HIT antibodies (26). We postulated that when PF4 is released in HIT, due to its cationic charge, it will bind to cell-free DNA (cfDNA) present in NETs and effect NET biology. To investigate PF4-NET interactions in real time, we studied neutrophils adherent to fibronectin-coated microfluidic channels stimulated with phorbol myristate acetate to release NETs. We confirmed that these cfDNA structures were NETs by using coimmunofluorescent staining to show that they contained citrullinated histones and MPO (Supplemental Figure 4). The NET-lined channels were then infused at venular flow rates with buffer containing PF4 at concentrations readily attained at sites of platelets activation $(5-100 \mu \mathrm{g} / \mathrm{ml})(14)$. Prior to exposure to PF4, NETs labeled with the cell membrane impermeant nucleic acid stain, SYTOX green, were round and transparent, with a cloud-like appearance 


\section{A}

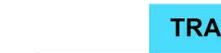

TRA

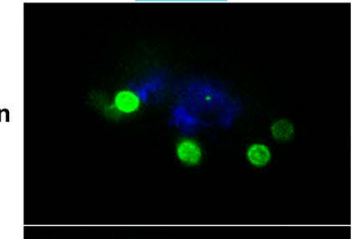

$2 \min$

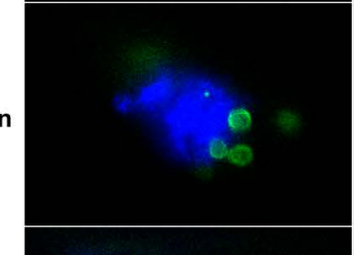

$5 \mathrm{~min}$

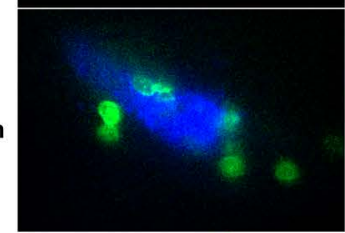

$60 \mathrm{~min}$
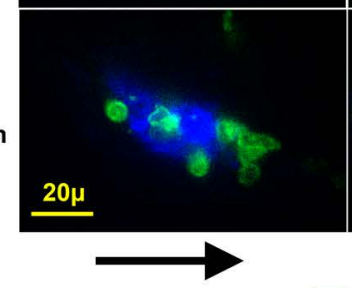

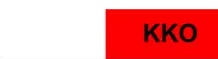

\section{KKO}

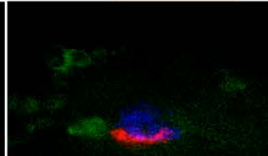

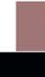
KKO + SCH527123

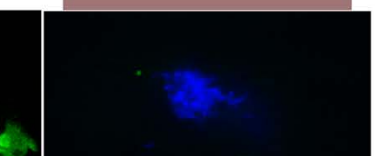

0

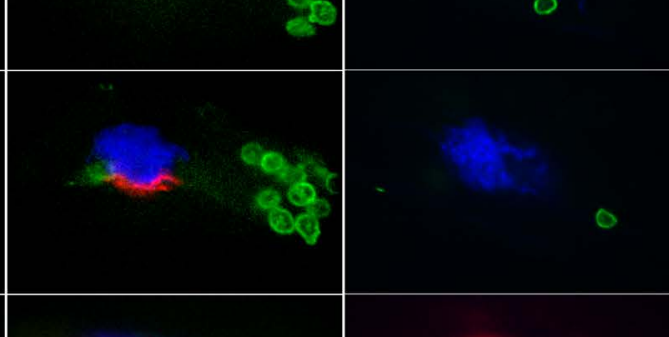

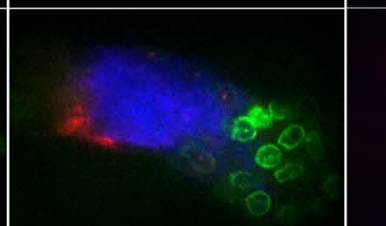

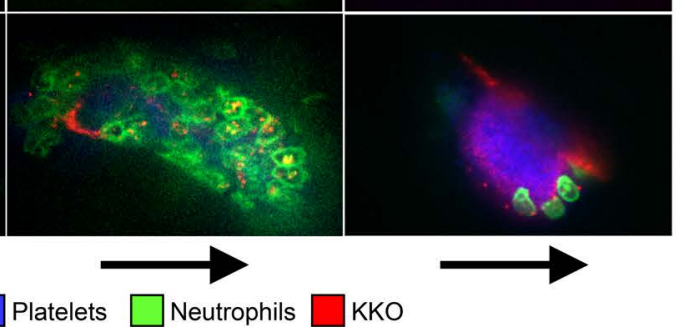

B

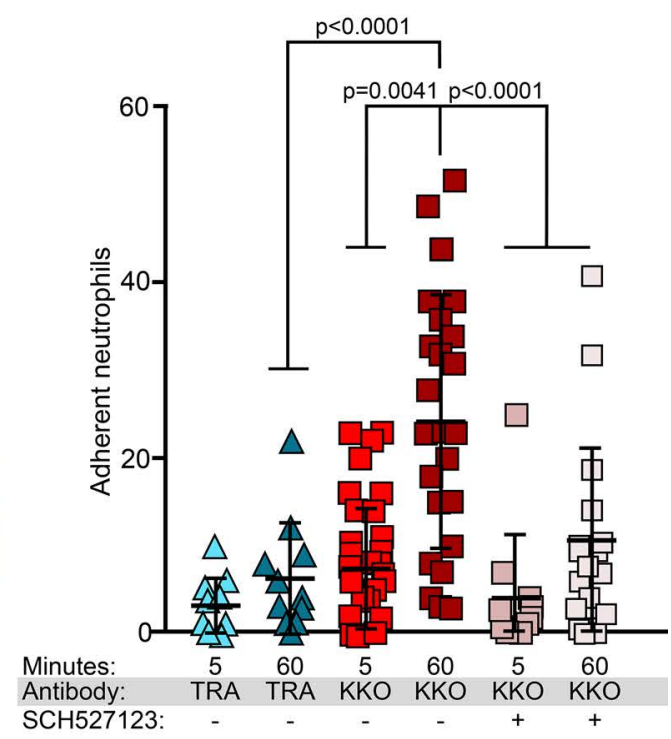

Figure 3. Chemokine dependency of neutrophil accumulation into venous thrombi in HIT. (A) Representative confocal images of cremaster venule injuries showing platelet (blue), neutrophil (green), and KKO staining (red) at 1-60 minutes following laser injury. Typical injuries are shown from mice infused with TRA, KKO, and KKO and SCH527123, a CXCR2 antagonist (60). An arrow indicating direction of flow is included. Scale bar: $20 \mu$. Same microscope and acquisition software as described in Figure 1D. (B) Quantification of adherent neutrophils in venule thrombi 5 and 60 minutes following laser injury. Thirty-two injuries were made in twelve KKO-treated mice. Seventeen injuries were made in three TRA-treated mice. Eleven injuries were made in three KKO plus SCH527123-treated mice. Individual data points and mean \pm 1 SD are shown. Comparative statistical analysis was performed by Kruskal-Wallis 1-way ANOVA.

that we termed "fluffy." When infused with HBSS (Thermo Fisher Scientific) or human plasma, the NETs extended, and when flow was stopped they rebounded to their former shape. Following treatment with either buffer or plasma containing PF4, the NETs became opaque and narrow with a sharply tapered tail that did not change shape when flow was stopped (Figure 4A, Supplemental Figure 4, and Supplemental Video 7). Maximum compaction, with a $50 \%$ reduction in NET area, was achieved when the NETs were flowed with HBSS containing PF 4 concentrations of $\geq 10 \mu \mathrm{g} / \mathrm{ml}$ (Figure 4B, for PF $4 \leq 25 \mu \mathrm{g} / \mathrm{ml}$ and not shown for $>25 \mu \mathrm{g} / \mathrm{ml}$ ). Confocal imaging of the compacted NETs, labeled with an anti-PF4 antibody and a fluorophore-conjugated secondary antibody, confirmed that PF4 bound to these structures (Figure 4A). Total NET DNA content measured by fluorescence intensity was preserved following PF4 infusion, excluding the possibility that the compacted NET appearance was due to the dissolution of DNA (Figure 4B). Coimmunofluorescent studies of NETs following PF4 infusion demonstrated that compaction does not lead to displacement of citrullinated histones or MPO (Supplemental Figure 4).

We next asked whether PF4-induced changes in NET morphology could effect NET behavior. While PF4-free NETs were highly susceptible to deoxyribonuclease (DNase) I at a concentration of $100 \mathrm{U} / \mathrm{ml}$, with near-complete digestion in $<2$ minutes, PF4-bound NETs were resistant to DNase I, with near-complete protection at PF4 concentrations of $\geq 10 \mu \mathrm{g} / \mathrm{ml}$ (Figure 4, C-E, and Supplemental Video 8). The infusion of human plasma containing PF4 was also found to confer resistance to DNase I-mediated digestion (Supplemental Figure 5), and similar results were observed for digestion with bacterial-derived micrococcal nuclease (Supplemental Figure 6). Comparable nuclease resistance was also observed in a static system not coated with fibronectin, showing that fibronectin, which has a DNA-binding domain and is known to interact with NETs (27), is not the cause of the observed changes in NET behavior (Supplemental Figure 5).

Previous in vitro studies reported that NETs are degraded by exposure to heparin (28), suggesting that NETs should be reduced in patients with recent heparin exposure. We found that at a therapeutic 

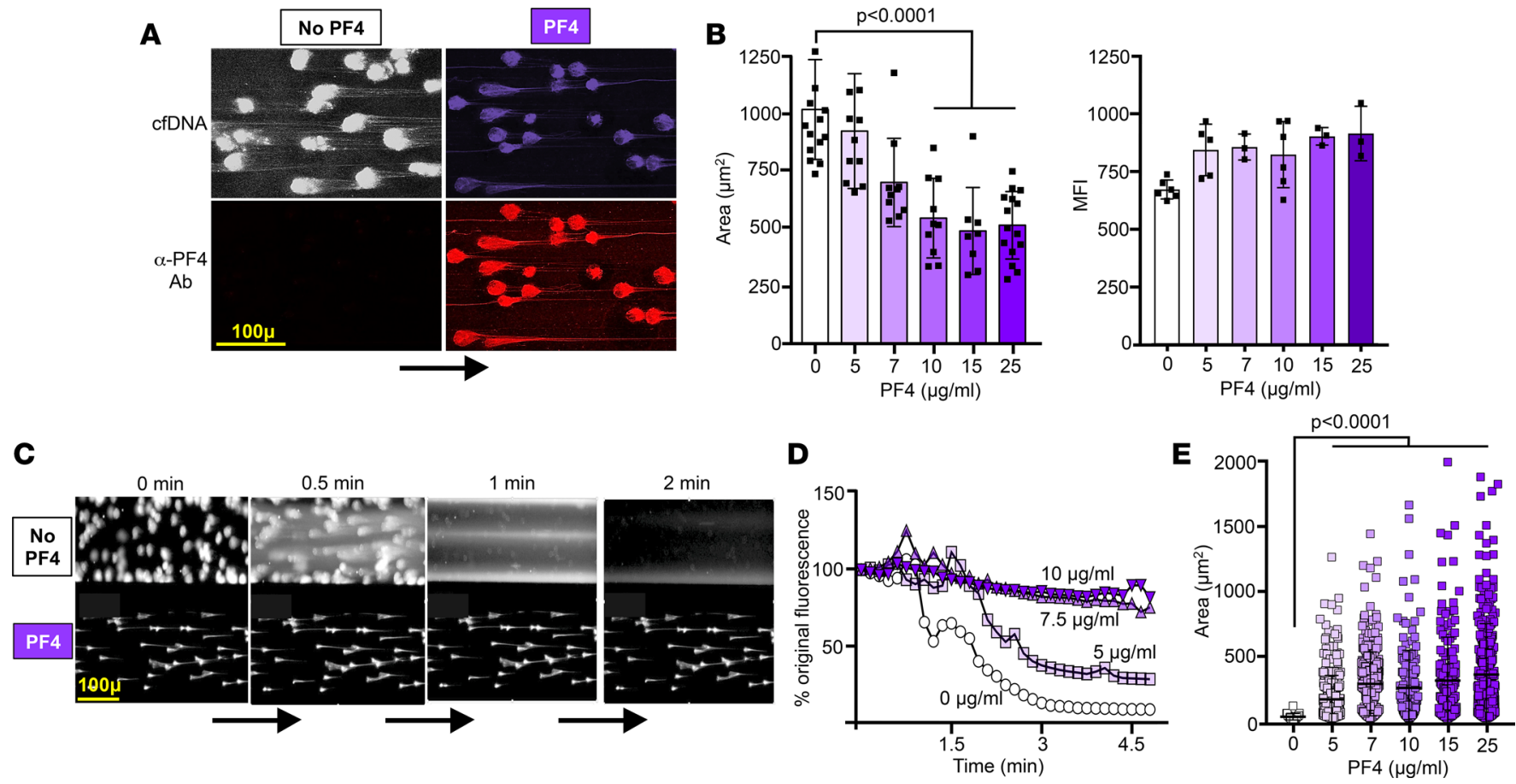

Figure 4. Microfluidic studies illustrating PF4-NET interactions. (A) Representative confocal images of NETs exposed to PF4 or buffer, demonstrating change in morphology. PF4 specifically adhered to the NET DNA, visualized by labeling with a polyclonal anti-PF4 antibody ( $\alpha$-PF4 Ab), causing the NETs to become compact. An arrow indicating direction of flow is included. Scale bar: $100 \mu$. Same microscope and acquisition software as in Figure $1 \mathrm{~A}$. (B) Mean \pm 1 SD of area covered by NETs exposed to the indicated PF4 concentration. NET area and mean fluorescent intensity (MFI) of SYTOX-labeled cfDNA are shown. $n=8-15$ channels per PF4 concentration, with visualization of 30-60 NETs per channel, was used in the NET area analysis. $n=3-6$ channels per PF4 concentration, with visualization of 30-60 NETs per channel, was used in the analysis of MFI. Comparative statistical analysis was performed by Kruskal-Wallis 1-way ANOVA. (C) Representative wide-field images of NETs with or without PF4 (10 $\mu \mathrm{g} / \mathrm{ml})$, simultaneously infused with DNase I (100 U/ml). Near-complete digestion of NETs not exposed to PF4 occurred within 2 minutes, whereas NETs compacted with PF4 remained intact. An arrow indicating direction of flow is included. Scale bar: $100 \mu$. (D) Graph showing representative changes in NET fluorescent intensity observed over a 5-minute infusion of DNase I $(100 \mathrm{U} / \mathrm{ml})$ in the presence of $0-10 \mu \mathrm{g} / \mathrm{ml} \mathrm{PF4}$. These studies were based on analysis of 3-4 channels for each condition, with 30-60 NETs observed in each channel. (E) Confocal images were taken of 3 channels per condition following digestion, and the residual mean volume \pm 1 SD of 30-100 NETs per channel was measured. Comparative statistical analysis was done by Kruskal-Wallis 1-way ANOVA.

concentration of $0.4 \mathrm{U} / \mathrm{ml}(29)$ or at a supratherapeutic concentration of 5,000 U/ml, heparin did not degrade NETs in our microfluidic system (Supplemental Video 9); however, we observed that exposure to heparin reversed compaction, with restoration of fluffy morphology (Supplemental Figure 7 , $A$ and B, and Supplemental Video 10). Confocal imaging of NETs labeled with a polyclonal anti-PF4 antibody confirmed that heparin treatment removed PF4 from the NETs (Supplemental Figure 7A), and DNase I infusion studies confirmed that decompaction of NETs restored their susceptibility to endonuclease digestion (Supplemental Figure 7, C and D). However, when NETs were exposed to 25 $\mu \mathrm{g} / \mathrm{ml}$ instead of $10 \mu \mathrm{g} / \mathrm{ml} \mathrm{PF} 4$, a concentration still within the range typically observed at sites of thrombosis (8), NET compaction and DNase resistance were maintained during coinfusion with therapeutic concentrations of heparin (Supplemental Figure 7, C and D).

In vitro effects of HIT antibodies on PF4-NET complexes. PF4 has previously been shown to form complexes with DNA aptamers that are HIT antigenic targets (30). We, therefore examined whether PF4NET complexes are also recognized by KKO and IgG isolated from HIT patient plasma using the microfluidic chambers to assess binding and function. We found that KKO and HIT IgG suspended in HBSS did not interact with noncompacted NETs but both bound to PF4-NET complexes (Figure 5A and Supplemental Figure 8). KKO dissolved in human plasma also bound specifically to PF4-NET complexes (Supplemental Figure 8). Antibody binding did not induce greater NET compaction, but it enhanced resistance to DNase I digestion (Figure 5B and Supplemental Video 11). This effect was found to be specific to HIT antibodies, as incubation with a polyclonal anti-PF4 antibody at $25 \mu \mathrm{g} / \mathrm{ml}$ did not provide similar protection from endonucleases (Figure 5, C and D). Similar results were seen using NETs immobilized to fibronectin-coated 96-well plates (Figure 5E) (31). 
A

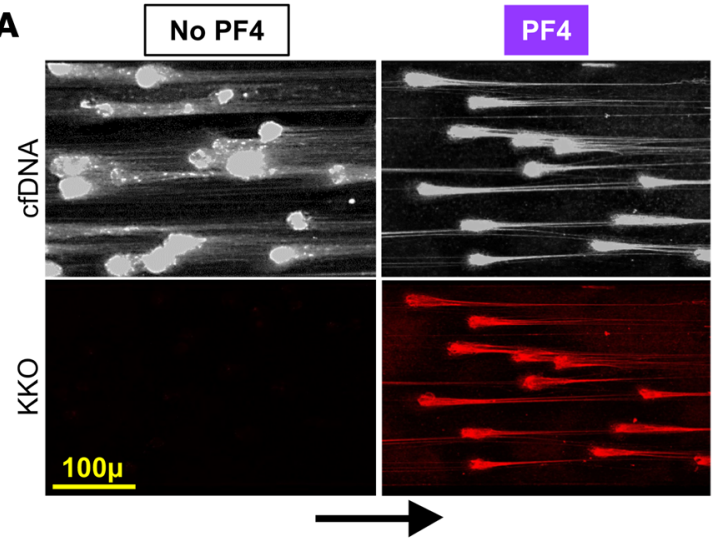

C

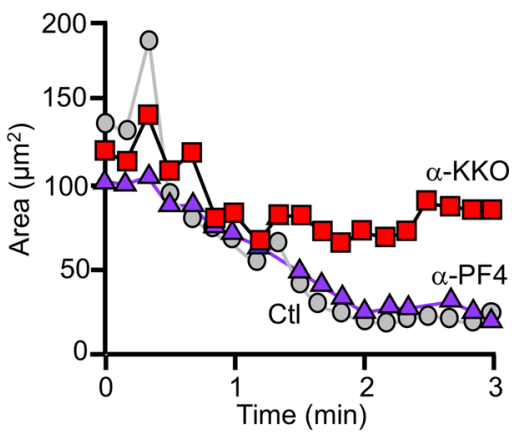

D

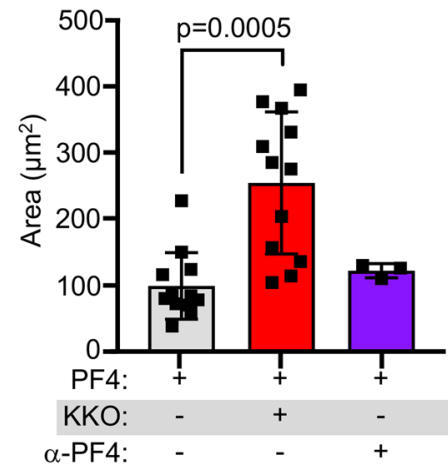

B

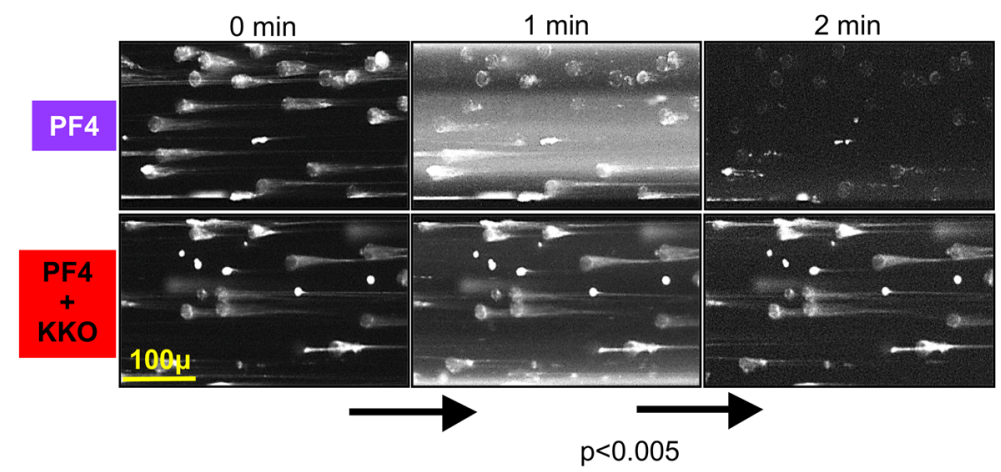

E

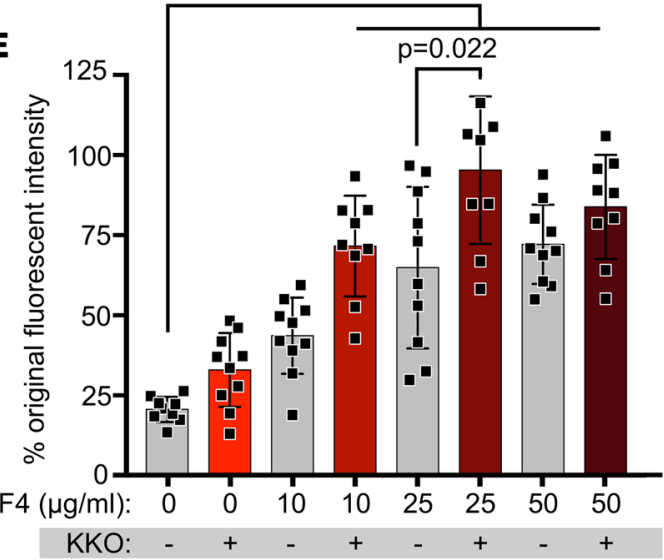

Figure 5. Microfluidic studies examining HIT antibody PF4-NET complex interactions. (A) Representative confocal images of activated neutrophils adherent to fibronectin-coated channels and stained for cfDNA (white) and KKO binding (red) in the absence and presence of $6.5 \mu \mathrm{g} / \mathrm{ml}$ PF4. An arrow indicating direction of flow is included. Scale bar: $100 \mu$. Same microscope and acquisition software as described in Figure 1A. (B) Representative wide-field images of adherent neutrophils, as in $\mathbf{A}$, but in the presence of $100 \mathrm{U} / \mathrm{ml}$ DNase1 and $6.5 \mu \mathrm{g} / \mathrm{ml} \mathrm{PF} 4$ with or without $25 \mu \mathrm{g} / \mathrm{ml} \mathrm{KKO}$ over 2 minutes. An arrow indicating direction of flow is included. Scale bar: $100 \mu$. (C) Representative graph showing the decrease in NET area over a 3-minute DNase I digestion. (D) Mean \pm 1 SD of the final area of NETs following a 5 -minute infusion of DNase I $(100 \mathrm{U} / \mathrm{ml})$ in the presence of $6.5 \mu \mathrm{g} / \mathrm{ml}$ PF4 and KKO or an anti-PF4 antibody ( $\alpha$ PF4), each at $25 \mu \mathrm{g} / \mathrm{ml} . n=12$ in the PF4- and the PF4 and KKO-treated arms. $n=3$ in the $\alpha$ PF4-treated arm. Comparative statistical analysis was performed by Kruskal-Wallis 1-way ANOVA. (E) PF4-induced resistance to DNase digestion under static conditions was measured using a previously described NET degradation assay (31). The graph shows the mean percent original fluorescent intensity $\pm 1 \mathrm{SD} . n=9-10$ per arm. Comparative statistical analysis between 3 or more groups was performed by Kruskal-Wallis 1-way ANOVA, and comparisons between 2 groups was performed with the Student's $t$ test.

Thrombosis in the HIT murine model in the presence or absence of PAD4. We then asked whether enhanced NET endonuclease resistance and the formation of PF4-NET-HIT antibody complexes, observed in vitro, contribute to in vivo thrombus development in the murine model of HIT. We hypothesized that the release of NETs by thrombus-adherent neutrophils and their subsequent modification by PF4 and HIT antibodies may play a role in the prothrombotic state that develops in these animals. To better understand the way in which NETosis may contribute to thrombosis in HIT, we studied Padi4 ${ }^{--}$HIT mice that are incapable of releasing NETs (Supplemental Figure 1). Previous studies have shown that Padi4-knockout mice do not release NETs and are protected from venous thrombosis (23). Following KKO injection, the severity of thrombocytopenia in Padi4 ${ }^{-/}$HIT mice was comparable to that seen in Padi4 $^{+/+}$HIT controls. (Figure 6A) The size of arteriolar thrombi following laser injury was unaffected by PAD4 deficiency (Figure 6B), and neutrophil incorporation into venule thrombi following TRA injection was similar in Padi4 ${ }^{-/-}$and $\mathrm{Padi4}^{+/+}$HIT mice. However, after KKO injection, the Padi4-/HIT mice developed smaller venular thrombi following injury (Figure 6, C and D), with incorporation of fewer neutrophils (Figure 6E). This difference became more pronounced with the passage of time (Figure 6C and Supplemental Video 11). Consistent with these studies in Padi4 ${ }^{-/}$HIT mice, Padi4 ${ }^{+/+}$ HIT mice treated with DNase I prior to KKO infusion had a significant decrease in platelet thrombus volume 60 minutes following injury (Figure 6C), suggesting that NETs play a role in thrombus growth and stabilization in this model. 
A

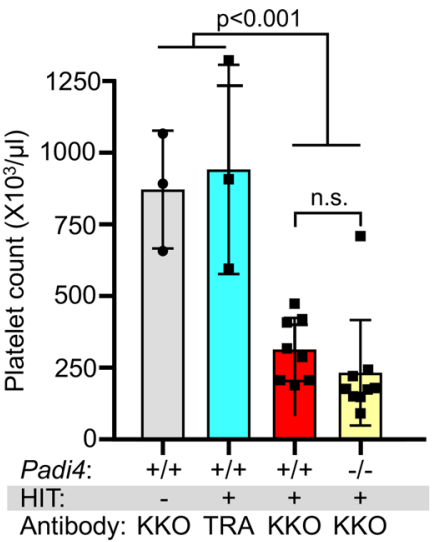

Antibody: KKO TRA KKO KKO

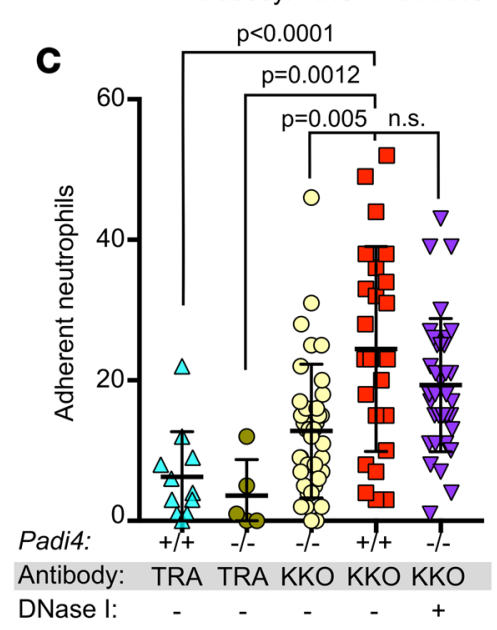

Padi4:
B

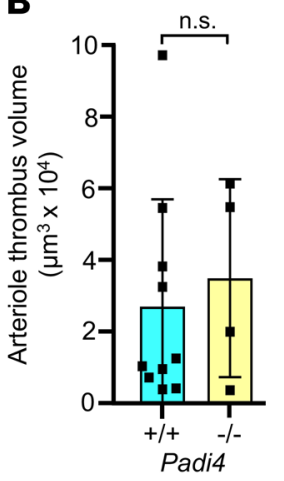

D

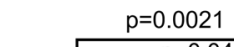

E
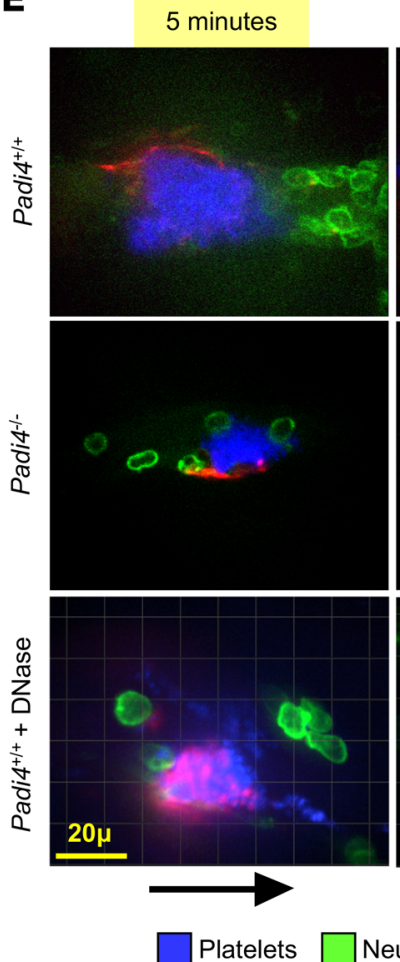

Platelets
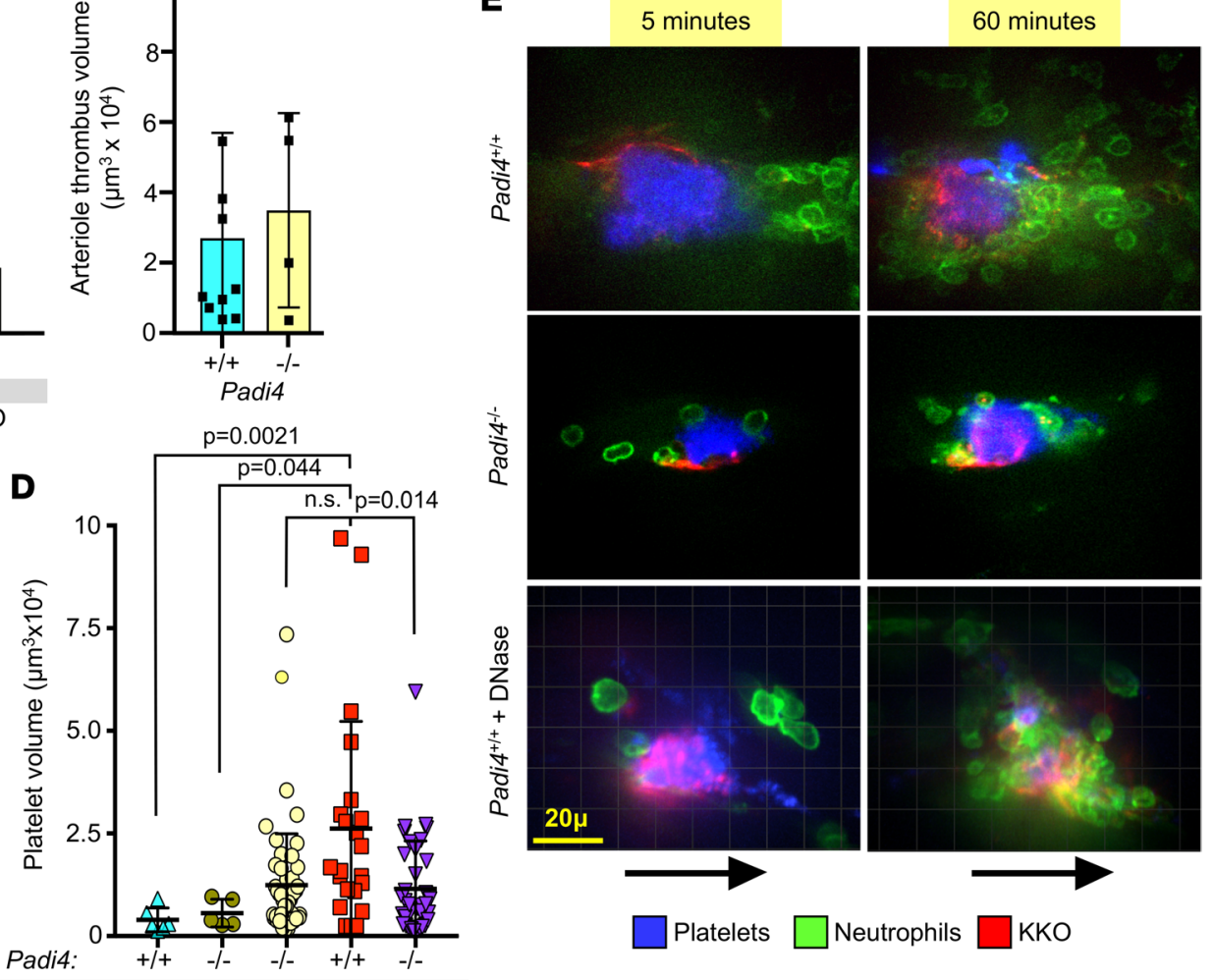

Antibody: TRA TRA KKO KKO KKO

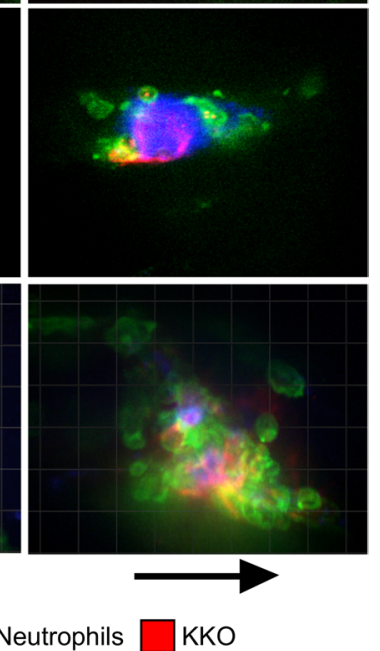

Figure 6. NETosis studies in the passive immunization Padi4-/- HIT mouse model. (A) Platelet counts 4 hours after intraperitoneal injection of KKO or TRA in HIT or Fc $\gamma \mathrm{RIIA}^{+}$, hPF4-/- ("HIT-") mice. Padi4 status is indicated. Mean \pm 1 SD is shown. Comparative statistical analysis was performed by Kruskal-Wallis 1-way ANOVA. (B) Same as in A, but arteriole thrombus size after KKO infusion in HIT mice with Padi4 status is indicated. Statistical analysis performed with a Student's $t$ test. (C and $\mathbf{D})$ The number of adherent neutrophils and the platelet volumes in cremaster venule thrombi in HIT mice 60 minutes after laser injury. Individual data points and mean \pm 1 SD are shown. 6 injuries were made in 3 TRA-treated HIT mice, 42 injuries were made in 10 KKO-treated Padi4 ${ }^{-1-}$ HIT mice, 5 injuries were made in 1 TRA-treated Padi4 ${ }^{-1-}$ mouse, 24 injuries were made in 13 KKO-treated HIT mice, and 37 injuries were made in 6 KKO-treated HIT mice following infusion of DNase I. Comparative statistical analysis was performed with a Kruskal-Wallis 1-way ANOVA. (E) Representative confocal images demonstrating the extent of platelet and neutrophil accumulation at the site of cremaster venule thrombi following HIT induction 5 and 60 minutes after the inciting laser injury and infusion of KKO in Padi4 ${ }^{/-}$mice compared with Padi4 ${ }^{+/+}$mice with or without DNase. An arrow indicating direction of flow is included. Scale bar: $20 \mu$. Same microscope and acquisition software as in Figure 1D.

Clinical indication of NETosis in HIT. It has been reported that circulating MPO levels are elevated in HIT (17), potentially implicating neutrophils and NETosis in the pathobiology of HIT. We confirmed this clinical finding comparing cfDNA and MPO-DNA complex levels in samples from patients at high or at low likelihood of having HIT (32), healthy controls, and patients with immune thrombocytopenia (ITP). cfDNA (Figure 7A) and MPO-DNA complex levels (Figure 7B) were significantly higher in individuals diagnosed with HIT compared with those in healthy controls and patients with an alternative cause of ITP. Although there was a trend toward higher MPO-DNA complex levels in HIT patients compared with ill patients evaluated for HIT, but found to have a low probability of having the disease, this finding did not reach statistical significance $(P=0.1)$, demonstrating that cfDNA release and NETosis occur in multiple proinflammatory/prothrombotic states that commonly occur in hospitalized patients.

\section{Discussion}

Over the past several years, it has become increasingly clear that the pathophysiology of HIT extends beyond platelet activation. It has previously been shown that HIT antibody interaction with other cells types, including the endothelium (11) and monocytes (10), leads to cell activation and a prothrombotic state. Others have also demonstrated that HIT antibodies activate PF4-exposed neutrophils through 
A

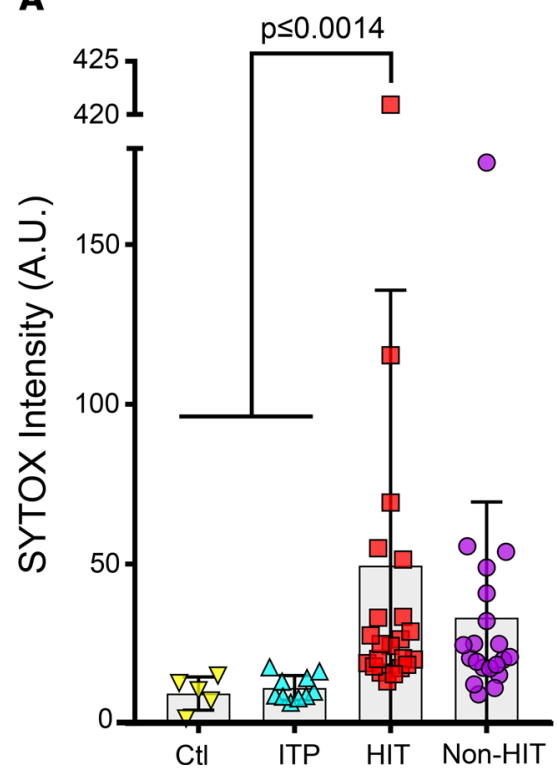

B

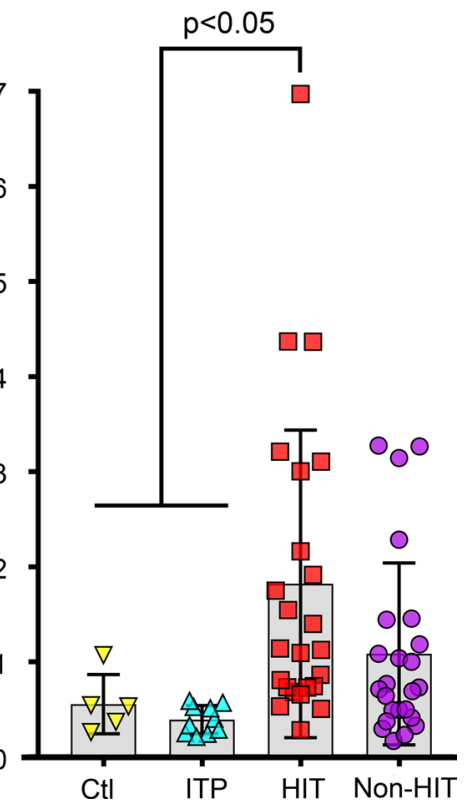

Figure 7. cfDNA and MPO-DNA levels in clinical samples. Measurements of (A) cfDNA levels and (B) MPO-DNA complex levels from normal controls (CtI), patients diagnosed with ITP, and patients evaluated for HIT that either had a confirmed diagnosis (HIT) or were unlikely to have HIT (Non-HIT). Individual measurements and mean \pm 1 SD are shown. Comparative statistical analysis was performed by Kruskal-Wallis 1-way ANOVA.

their FcrRIIa receptors, leading to increased expression of Mac-1 on the cell surface and enhanced neutrophil aggregate formation (14). In this paper, we explore specific pathways by which neutrophils may contribute to thrombosis in HIT and propose that they do so through multiple steps, including the stimulation of neutrophil adhesion to inflamed endothelium, the promotion of neutrophil migration into venular thrombi, and the formation of antigenic PF4-NET-HIT antibody complexes.

The basis for enhanced binding of HIT-activated neutrophils to the endothelium in large vessels and the microcirculation requires further exploration. Prior studies have shown that HIT antibody exposure increases neutrophil surface expression of Mac-1 (14). Endothelial cells are also activated by PF4 and HIT antibodies, increasing surface expression of $\mathrm{P}$ and $\mathrm{E}$ selectins (33). Our finding of enhanced in vitro neutrophil binding parallels the increased adhesion seen in vivo in the setting of our murine model of HIT, with or without endothelial cell injury. We speculate that neutrophil adhesion immediately downstream of growing venular injury may be due to increased turbulent flow around larger thrombi that is known to enhance neutrophil adhesion to the endothelial lining (34). It may also be due to the release of PF4 from activated platelets within the thrombus that results in increased assembly of antigenic complexes on the downstream endothelium, leading to more HIT antibody binding and endothelial activation (11). The role of each of these mechanisms in the accumulation of neutrophils in injured venules requires additional investigation to identify opportunities for intervention.

After adhering to the endothelium downstream of thrombosis, many neutrophils migrate in a retrograde manner and are incorporated into the venular clots. Retrograde migration may occur in response to a chemotactic gradient emanating from degranulating platelets. A similar process, termed "directed intravascular migration," has been described in ischemia/reperfusion injuries, in which degranulating platelets release CXCL7 (neutrophil-activating peptide 2 [NAP2]) and CXCL5 (epithelial-derived neutrophil-activating peptide 78 [ENA-78]), leading to CXCR2-dependent neutrophil recruitment (35). The retrograde migration we observed in venular HIT thrombi is also similar to "neutrophil swarming," a process in which large numbers of neutrophils rapidly accumulate at sites of infection or sterile injury where they release NETs (36). In support of this hypothesis, we show that the retrograde migration of neutrophils into venous thrombi can be abrogated with the blockade of CXCR2. Whether NAP2 and/ or ENA-78, both released from platelets and bound by CXCR2 (37), contribute to the chemogradient remains to be determined. Further investigation needs to be done to ascertain if this pattern of adhesion occurs in other prothrombotic disorders associated with venous thrombosis. 
Many groups have proposed that NET release links neutrophil accumulation to thrombus progression $(23,38,39)$. We posit that NETs released in HIT are uniquely prothrombotic because the binding of PF4 and HIT antibodies causes them to become immunogenic. Previous studies have shown that nucleic acid binding leads to the exposure of the same antigenic epitopes present on PF4/heparin complexes (30). Mice injected with PF4/nucleic acid complexes develop antibodies that cross-reacted with PF4/ heparin complexes (30). Our microfluidic channel experiments support that PF4 forms complexes with chromatin DNA in the same way as PF4 binds heparin to form macroaggregates (40). Our studies showing that HIT IgG isolated from human plasma binds to these PF4-NET complexes confirm that they contain HIT antigenic epitopes.

In addition to producing HIT antigenic complexes, we have observed that PF4 binding leads to chromatin compaction that causes NETs to become DNase resistant. It has previously been shown that NET binding to the cationic peptide LL-37 leads to enhanced protection from nuclease digestion (41), in a manner similar to what we have observed with PF4. While DNase-resistant NETs may have increased antimicrobial activity, impaired NET degradation has been implicated in the pathogenesis of autoimmune diseases, including antiphospholipid antibody syndrome and lupus $(42,43)$. In these disorders, DNase resistance is thought to occur due to the development of anti-NET immunoglobulins that interfere with DNase binding to cfDNA (31). In HIT, PF4 forms complexes with NETs that are bound by HIT antibodies that provide additional protection from DNases. These stable, compacted PF4-NET-HIT IgG aggregates then serve as a Fc-rich surface that can recruit and activate circulating hematopoietic cells via their Fc $\gamma$ RIIa receptors and activate the complement cascade (15).

To test this hypothesis, we generated Padi4 ${ }^{-1}$ HIT mice that do not undergo NETosis $(20,23,44)$. In these animals, venular thrombi generated after HIT induction are markedly smaller than those observed in animals capable of NET release, with fewer incorporated neutrophils and decreased platelet volumes. This suggests that NETs contribute to the early stages of venule thrombus growth, enhancing both platelet accumulation and neutrophil recruitment. This effect was not observed in laser injury-induced arteriole thrombi, which have little neutrophil infiltration irrespective of PAD4 activity. Moreover, the loss of PAD4 activity — and likely NET formation - did not affect the severity of thrombocytopenia in HIT mice, suggesting that the development of venular thrombi is unrelated to the mechanism underlying the development of thrombocytopenia.

We next treated HIT mice with DNase I prior to cremaster laser injury to determine if NETs functionally contribute to thrombus formation in our model and found that this led to a significant decrease in cremaster venule thrombus platelet volumes. This finding further supports our hypothesis that NETs play a role in thrombus growth and stabilization in this model. However, unlike in the Padi4 ${ }^{-/-}$HIT mice, the number of thrombus-adherent neutrophils did not fall in accordance with the decrease in platelet volumes. This difference may occur because Padi4 ${ }^{-1}$ mice do not release NETs, whereas PF4-compacted NETs are incompletely degraded by DNase and may still expose PF4-NET-HIT IgG aggregates that can recruit circulating neutrophils. Nonetheless, the decrease in venular thrombus size raises the possibility that DNase treatment may be effective in preventing the venular prothrombotic state in HIT.

In summary, we demonstrate that neutrophils contribute to the prothrombotic state in HIT through enhanced adhesion to the endothelium downstream of venous thrombi and subsequent retrograde migration into these thrombi. There, activated neutrophils release NETs that form complexes with PF4 that are subsequently bound by HIT antibodies. These PF4-NET-HIT antibody complexes are protected from DNases and may contribute to thrombus development by exposing an Fc-rich surface. Thus, our studies extend the range of PF4-bound HIT antigenic polyanionic targets beyond heparin, GAGs, and polyphosphates. HIT mice that have neutrophils unable to undergo NETosis have smaller venous thrombi but still develop enhanced arterial clots and thrombocytopenia. Further work is required to understand whether neutrophils contribute similarly to the development of venous thrombi in other immune/inflammatory prothrombotic states, such as antiphospholipid antibody syndrome and sepsis. More investigation is needed to determine if blocking these pathways will help prevent venous thromboembolic complications in patients with HIT or these other prothrombotic disorders.

\section{Methods}

Mice and human samples. HIT mice were transgenic for both platelet-specific $\mathrm{hPF} 4\left(\mathrm{hPF} 4^{+}\right)$and human Fc $\gamma$ RIIa $\left(\mathrm{Fc}_{\mathrm{RIIa}}\right)$ (21). All HIT mice were also on a $C x c l 4^{-/-}$background (45), as murine PF4 is not recognized by HIT antibodies (46). Genetic alterations were confirmed by PCR analyses (47). We used 
the CRISPR/Cas9 genome editing system (48) to disrupt Padi4 exon2 in HIT mice (Padi4 ${ }^{-/}$HIT mice, Supplemental Figure 1A). Guide RNAs flanking the targeted exon were designed to minimize off-target interactions (49) (5'-CCTAAGGGCTACACAACCTT-3' and 5'-GCTGGCTGCTTTCACCTGTAC-3') and injected into harvested embryos at concentrations of $50 \mathrm{ng} / \mu 1$ along with Cas $9 \mathrm{mRNA} 100 \mathrm{ng} / \mu 1 \mathrm{at}$ the Children's Hospital of Philadelphia (CHOP). Oligonucleotide primers designed to amplify the 0.2$\mathrm{kb}$ region of the gene-encasing exon 2 (5'-CATCTGTTCTGCTGCTGGCTG-3' and 5'-CTCCTAAGGGCTACACAACCTTC-3') were used to confirm the presence of the desired gene disruption. Functional abrogation of PAD4 activity was confirmed by stimulating neutrophils isolated from the bone marrow of Padi4 $^{-1}$ mice and plated on poly-L-lysine-coated slides (MilliporeSigma) with $100 \mathrm{nM}$ phorbol-12 myristate 13 -acetate (MilliporeSigma) overnight at $37^{\circ} \mathrm{C}$ and then staining the cells with SYTOX green $(1 \mu \mathrm{M})$ and anti-citrullinated histone $\mathrm{H} 3$ antibody (anti-citH3, Ab5103, MilliporeSigma) at a dilution of 1:500 to visualize NET release (Supplemental Figure 1C). Both Padi4 $^{+/+}$HIT and Padi4 ${ }^{-1}$ HIT mice were studied at 10-30 weeks of age. Only male mice were used in the cremaster vessel injury experiments; however, in previous studies, we have not appreciated any sex differences in thrombosis in the passive immunization HIT murine model following photochemical carotid artery injury (10).

Deidentified plasma samples were obtained from patients with suspected HIT (50). All subjects were evaluated with a polyspecific PF4/heparin ELISA (Immucor) (19) and a serotonin-release assay (51, 52) and classified as having or not having HIT by a panel of 3 independent expert adjudicators who based their decision on laboratory results and clinical findings. Control plasma was obtained from healthy individuals and patients diagnosed with ITP (53). Citrated plasma was stored at $-80^{\circ} \mathrm{C}$, and MPO-DNA complex levels and cfDNA levels were also measured as previously described (54). IgG was isolated from plasma from HIT patients and healthy donors using protein G agarose (Pierce) (8).

NET-lined microfluidic channel studies. Experiments were performed using a BioFlux 200 Controller (Fluxion) as described previously (55). The BioFlux channels were visualized with an Axio Observer Z1 inverted microscope (Zeiss) equipped with a motorized stage and an HXP-120 C metal halide illumination source. The microscope and image acquisition were controlled by BioFlux Montage software with a MetaMorph-based platform (Molecular Devices). Isolated human neutrophils $\left(2 \times 10^{6} \mathrm{cells} / \mathrm{ml}\right)$ were incubated with $1 \mathrm{ng} / \mathrm{ml} \mathrm{TNF- \alpha} \mathrm{(Gibco)}$ at room temperature for 10 minutes and then flowed through channels coated with $50 \mu \mathrm{g} / \mathrm{ml}$ fibronectin (MilliporeSigma), to which they adhered, and then incubated with $100 \mathrm{ng} / \mathrm{ml}$ phorbol myristate acetate overnight at $37^{\circ} \mathrm{C}$. cfDNA release was visualized with $1 \mu \mathrm{M}$ SYTOX green or orange, and the channels were infused with recombinant PF4 $(0-200 \mu \mathrm{g} / \mathrm{ml})$, expressed, and purified as we described previously (56), with or without unfractionated porcine heparin $\left(0.4 \mathrm{U} / \mathrm{ml}, \mathrm{BD}\right.$ Biosciences) at $2-5$ dynes $/ \mathrm{cm}^{2}$. To confirm that the cfDNA structures adherent to the microfluidic channel walls were NETs, we performed coimmunolocalization in which channels with or without PF4 $(25 \mu \mathrm{g} / \mathrm{ml})$ were incubated with either anti-citH3 at $4^{\circ} \mathrm{C}$ overnight or anti-MPO at $37^{\circ} \mathrm{C}$ for 1 hour and then incubated with an Alexa Fluor 594-conjugated secondary antibody for 1 hour at $37^{\circ} \mathrm{C}$ prior to imaging with a Zeiss LSM 710 laser-scanning confocal microscope. Structures with colocalization of cfDNA and MPO or citrullinated histones were deemed to be NETs. PF4-NET complexes were then incubated with $25 \mu \mathrm{g} / \mathrm{ml} \mathrm{KKO}$, a HIT-like monoclonal antibody (19), or a polyclonal antiPF4 antibody (Abcam, Ab9561) for 1 hour at $37^{\circ} \mathrm{C}$. NET digestion studies were carried out by infusing the channels with $100 \mathrm{U} / \mathrm{ml}$ DNase I (MilliporeSigma), at 2 dynes $/ \mathrm{cm}^{2}$, after which the channels were washed with PBS (Gibco) and fixed with 2\% paraformaldehyde (BD Biosciences). To avoid laser-induced changes to the conformation of DNA (57), we minimized laser exposure during our experiments. To account for this effect when studying whether KKO binding to PF4-NET complexes increased resistance to DNase, we compared NET digestion by analyzing videos in which channels with and without KKO were included in the same visual field and exposed to UV light for the same amount of time. Channels were blocked with PBS with 2\% BSA (MilliporeSigma), and PF4-NET complexes were visualized by incubating SYTOX-labeled NETs with a rabbit anti-human PF4 antibody at $37^{\circ} \mathrm{C}$ for 1 hour, rinsing the channels with PBS flowed at 5 dynes $/ \mathrm{cm}^{2}$ for 5 minutes, and then incubating with a goat anti-rabbit secondary antibody (Abcam, Ab150080) at $37^{\circ} \mathrm{C}$ for 1 hour. KKO was labeled with Alexa Fluor 647 (Thermo Fisher Scientific) prior to NET channel infusion. NET complexes were imaged with a Zeiss LSM 710 laser-scanning confocal microscope. Similar studies were done using $100 \mathrm{U} / \mathrm{ml}$ bacterial-derived micrococcal nuclease (New England Biolabs). Data were analyzed using ImageJ (NIH) open-souce image processing software (58). 
Endothelialized channel microfluidic studies. HUVECs (Lonza) at passage 3-4 (5 × $10^{6}$ cells) were seeded onto fibronectin-coated (50 $\mathrm{g} / \mathrm{ml}$, MilliporeSigma) channels of 48-well BioFlux plates (Fluxion), allowed to adhere, and then cultured at $37^{\circ} \mathrm{C}$ under $5 \% \mathrm{CO}_{2}$ in endothelial cell growth media (Lonza) for 2-3 days to become confluent. HUVECs were incubated with TNF- $\alpha(1-10 \mathrm{ng} / \mathrm{ml})$ to simulate inflammation. Whole blood samples obtained from healthy human donors were fluorescently labeled with $2 \mathrm{mM}$ calcein AM to visualize leukocytes and platelets and then incubated with KKO or TRA, an isoimmune monoclonal control $(25 \mu \mathrm{g} / \mathrm{ml})$, for 15 minutes. The samples were then recalcified with $\mathrm{CaCl}_{2}$ (11 mM final concentration) and flowed through the channels at 5 dynes $/ \mathrm{cm}^{2}$. When using ImageJ to count endothelial-adherent cells, size exclusion thresholds were used to distinguish platelets from leukocytes. To assess KKO binding to TNF- $\alpha-$ stimulated endothelium, the HUVEC-lined channels were infused with PBS containing PF4 (10 $\mu \mathrm{g} / \mathrm{ml})$ and $\mathrm{KKO}(25 \mu \mathrm{g} / \mathrm{ml})$ conjugated with Alexa Fluor 488 (Invitrogen) at $5 \mathrm{dynes} / \mathrm{cm}^{2}$. Following the infusion, the channels were washed with PBS and fixed with $2 \%$ paraformaldehyde (BD Biosciences). KKO binding was quantified based on fluorescent measurements obtained from a Zeiss LSM 710 laser-scanning confocal microscope. Levels of KKO-endothelial binding were compared with those observed after the whole blood samples containing PF4 and KKO were infused through HUVEC channels that had been subjected to hematoporphyrin (50 $\mu \mathrm{g} / \mathrm{ml}$, MilliporeSigma) photochemical injury, as our group has previously described (11).

Femoral vein neutrophil-rolling studies. HIT mice were anesthetized with Nembutal and a skin flap extending from the ankle to thigh was removed to expose the femoral vein. SYTOX orange $(2 \mathrm{nM})$ and $\mathrm{F}\left(\mathrm{ab}^{\prime}\right)_{2}$ fragments $(0.2 \mathrm{mg} / \mathrm{g}$ mouse) directed against Ly6-G and CD41 were infused as $100-\mu 1$ boluses via a catheter placed into the jugular vein to detect cfDNA, neutrophils, and platelets, respectively. Confocal microscopy was used as described previously (59) to obtain videos of neutrophil rolling and adhesion in the femoral vein at baseline and 10 minutes after $1 \mu \mathrm{g} / \mathrm{g}$ KKO was infused via the jugular catheter. Data were collected and confocal time-lapsed images were analyzed using Slidebook 6.0 (Intelligent Imaging Innovations) to compare neutrophil behavior before and after KKO exposure.

Supplemental Methods. Please see the Supplemental Methods for a list of antibodies and other labeled probes as well as details regarding the isolation of human neutrophils, bioassay for NET digestion, glassslide NET compaction and digestion studies, and a description of cremaster laser arteriole and venule injury studies in the setting of the passive immunization murine model of HIT.

Statistics. Differences between 2 groups were compared using a 2-tailed Student's $t$ test with Welsh's correction or a Mann-Whitney $U$ test. Normal distribution was tested using the D'Agostino and Pearson normality test. Differences between $>2$ groups were determined by 1- or 2-way ANOVA (as appropriate), and a Kruskal-Wallis test was performed when data was not normally distributed. Multiplicity-corrected $P$ values are reported for multiple comparisons. Statistical analyses were performed using Microsoft Excel 2011 and GraphPad Prism 7.0 (GraphPad Software). Differences were considered statistically significant when $P$ values were $\leq 0.05$.

Study approval. Animal procedures were approved by the Institutional Animal Care and Use Committee at CHOP in accordance with NIH guidelines and the Animal Welfare Act. Anonymized human blood was collected after signed, informed consent was provided by healthy donors, and approval for studies using human blood was obtained from CHOP's Human Review Board in accordance with Declaration of Helsinki Principles.

\section{Author contributions}

KG carried out and evaluated these studies and prepared the first draft and subsequent revisions of this manuscript. MK and IJ assisted on many of the microfluidic experiments. VH assisted on most of the in situ studies. JW and MK developed the femoral vein visualization protocol and assisted in its performance. MPL and AC provided the clinically evaluated and interpreted samples. DBC, LR, MAK, and GMA provided research guidance. MP provided overall project organization and direction, data interpretation, and manuscript preparation.

\section{Acknowledgments}

This work was supported by grants from the National Heart, Lung, and Blood Institute (P01HL110860 to MP, GMA, DBG, and LR; K23HL112903 to AC; T32HL007150 to KG); grants from the NIH (R01HL136512 to GMA, R01HL14122 to DBC and MP, and R01HL139448 to MP and DBC); and by an American Society of Hematology Fellow Research Award (to KG). We thank Steven E. McKenzie for human Fc $\gamma$ RIIa $\left(F c \gamma R I I a^{+}\right)$ mice, and we thank Kristin M. Hudock and G. Scott Worthen for insightful discussions. 
Address correspondence to: Mortimer Poncz, Children's Hospital of Philadelphia, 3615 Civic Center Boulevard, ARC, Room 317, Philadelphia, Pennsylvania 19104, USA. Phone: 215.590.3574; Email: poncz@email.chop.edu.

1. Nand S, Wong W, Yuen B, Yetter A, Schmulbach E, Gross Fisher S. Heparin-induced thrombocytopenia with thrombosis: incidence, analysis of risk factors, and clinical outcomes in 108 consecutive patients treated at a single institution. Am J Hematol. 1997;56(1):12-16

2. Wallis DE, Workman DL, Lewis BE, Steen L, Pifarre R, Moran JF. Failure of early heparin cessation as treatment for heparin-induced thrombocytopenia. Am J Med. 1999;106(6):629-635.

3. Kelton JG, Hursting MJ, Heddle N, Lewis BE. Predictors of clinical outcome in patients with heparin-induced thrombocytopenia treated with direct thrombin inhibition. Blood Coagul Fibrinolysis. 2008;19(6):471-475.

4. Linkins LA, et al. Treatment and prevention of heparin-induced thrombocytopenia: Antithrombotic Therapy and Prevention of Thrombosis, 9th ed: American College of Chest Physicians Evidence-Based Clinical Practice Guidelines. Chest. 2012;141(2 Suppl):e495S-e530S.

5. Zhou A, et al. Is the incidence of heparin-induced thrombocytopenia affected by the increased use of heparin for VTE prophylaxis? Chest. 2012;142(5):1175-1178.

6. Cervera R. Antiphospholipid syndrome. Thromb Res. 2017;151 Suppl 1:S43-S47.

7. Donzé JD, Ridker PM, Finlayson SR, Bates DW. Impact of sepsis on risk of postoperative arterial and venous thromboses: large prospective cohort study. BMJ. 2014;349:g5334.

8. Rauova L, Zhai L, Kowalska MA, Arepally GM, Cines DB, Poncz M. Role of platelet surface PF4 antigenic complexes in heparin-induced thrombocytopenia pathogenesis: diagnostic and therapeutic implications. Blood. 2006;107(6):2346-2353.

9. Rauova L, Arepally G, McKenzie SE, Konkle BA, Cines DB, Poncz M. Platelet and monocyte antigenic complexes in the pathogenesis of heparin-induced thrombocytopenia (HIT). J Thromb Haemost. 2009;7 Suppl 1:249-252.

10. Rauova L, et al. Monocyte-bound PF4 in the pathogenesis of heparin-induced thrombocytopenia. Blood. 2010;116(23):5021-5031.

11. Hayes V, et al. Endothelial antigen assembly leads to thrombotic complications in heparin-induced thrombocytopenia. J Clin Invest. 2017;127(3):1090-1098.

12. Arepally GM. Heparin-induced thrombocytopenia. Blood. 2017;129(21):2864-2872.

13. Poncz M. Mechanistic basis of heparin-induced thrombocytopenia. Semin Thorac Cardiovasc Surg. 2005;17(1):73-79.

14. Xiao Z, Visentin GP, Dayananda KM, Neelamegham S. Immune complexes formed following the binding of anti-platelet factor 4 (CXCL4) antibodies to CXCL4 stimulate human neutrophil activation and cell adhesion. Blood. 2008;112(4):1091-1100.

15. Qiao J, Al-Tamimi M, Baker RI, Andrews RK, Gardiner EE. The platelet Fc receptor, FcyRIIa. Immunol Rev. 2015;268(1):241-252.

16. Mayadas TN, Tsokos GC, Tsuboi N. Mechanisms of immune complex-mediated neutrophil recruitment and tissue injury. Circulation. 2009;120(20):2012-2024.

17. Khairy M, et al. Polymorphonuclear leukocyte and monocyte activation induced by plasma from patients with heparin-induced thrombocytopenia in whole blood. Thromb Haemost. 2004;92(6):1411-1419.

18. Martinod K, Wagner DD. Thrombosis: tangled up in NETs. Blood. 2014;123(18):2768-2776.

19. Arepally GM, et al. Characterization of a murine monoclonal antibody that mimics heparin-induced thrombocytopenia antibodies. Blood. 2000;95(5):1533-1540.

20. Li P, Li M, Lindberg MR, Kennett MJ, Xiong N, Wang Y. PAD4 is essential for antibacterial innate immunity mediated by neutrophil extracellular traps. $J$ Exp Med. 2010;207(9):1853-1862.

21. Reilly MP, et al. Heparin-induced thrombocytopenia/thrombosis in a transgenic mouse model requires human platelet factor 4 and platelet activation through FcgammaRIIA. Blood. 2001;98(8):2442-2447.

22. Chapman RW, et al. A novel, orally active CXCR1/2 receptor antagonist, Sch527123, inhibits neutrophil recruitment, mucus production, and goblet cell hyperplasia in animal models of pulmonary inflammation. J Pharmacol Exp Ther. 2007;322(2):486-493.

23. Brill A, et al. Neutrophil extracellular traps promote deep vein thrombosis in mice. J Thromb Haemost. 2012;10(1):136-144.

24. Jenne CN, Urrutia R, Kubes P. Platelets: bridging hemostasis, inflammation, and immunity. Int J Lab Hematol. 2013;35(3):254-261.

25. McDonald B, Urrutia R, Yipp BG, Jenne CN, Kubes P. Intravascular neutrophil extracellular traps capture bacteria from the bloodstream during sepsis. Cell Host Microbe. 2012;12(3):324-333.

26. Cines DB, et al. Heparin-induced thrombocytopenia: an autoimmune disorder regulated through dynamic autoantigen assembly/disassembly. J Clin Apher. 2007;22(1):31-36.

27. Monti M, et al. Integrin-dependent cell adhesion to neutrophil extracellular traps through engagement of fibronectin in neutrophil-like cells. PLoS One. 2017;12(2):e0171362.

28. Fuchs TA, et al. Extracellular DNA traps promote thrombosis. Proc Natl Acad Sci USA. 2010;107(36):15880-15885.

29. Brill-Edwards P, Ginsberg JS, Johnston M, Hirsh J. Establishing a therapeutic range for heparin therapy. Ann Intern Med. 1993;119(2):104-109.

30. Jaax ME, et al. Complex formation with nucleic acids and aptamers alters the antigenic properties of platelet factor 4. Blood. 2013;122(2):272-281.

31. Hakkim A, et al. Impairment of neutrophil extracellular trap degradation is associated with lupus nephritis. Proc Natl Acad Sci USA. 2010;107(21):9813-9818.

32. Cuker A. Update in the diagnosis and management of heparin-induced thrombocytopenia. Clin Adv Hematol Oncol. 2012;10(7):453-455

33. Blank M, et al. Anti-platelet factor 4/heparin antibodies from patients with heparin-induced thrombocytopenia provoke direct activation of microvascular endothelial cells. Int Immunol. 2002;14(2):121-129.

34. Begandt D, Thome S, Sperandio M, Walzog B. How neutrophils resist shear stress at blood vessel walls: molecular mechanisms, subcellular structures, and cell-cell interactions. J Leukoc Biol. 2017;102(3):699-709. 
35. Ghasemzadeh $\mathrm{M}$, et al. The CXCR1/2 ligand NAP-2 promotes directed intravascular leukocyte migration through platelet thrombi. Blood. 2013;121(22):4555-4566.

36. Kienle K, Lämmermann T. Neutrophil swarming: an essential process of the neutrophil tissue response. Immunol Rev. 2016;273(1):76-93.

37. Chapman RW, Phillips JE, Hipkin RW, Curran AK, Lundell D, Fine JS. CXCR2 antagonists for the treatment of pulmonary disease. Pharmacol Ther. 2009;121(1):55-68.

38. Wolach O, et al. Increased neutrophil extracellular trap formation promotes thrombosis in myeloproliferative neoplasms. $\mathrm{Sci}$ Transl Med. 2018;10(436):eaan8292.

39. Dyer MR, et al. Deep vein thrombosis in mice is regulated by platelet HMGB1 through release of neutrophil-extracellular traps and DNA. Sci Rep. 2018;8(1):2068.

40. Brandt S, et al. Polyphosphates form antigenic complexes with platelet factor 4 (PF4) and enhance PF4-binding to bacteria. Thromb Haemost. 2015;114(6):1189-1198.

41. Neumann A, et al. Novel role of the antimicrobial peptide LL-37 in the protection of neutrophil extracellular traps against degradation by bacterial nucleases. J Innate Immun. 2014;6(6):860-868.

42. Leffler J, Stojanovich L, Shoenfeld Y, Bogdanovic G, Hesselstrand R, Blom AM. Degradation of neutrophil extracellular traps is decreased in patients with antiphospholipid syndrome. Clin Exp Rheumatol. 2014;32(1):66-70.

43. Leffler J, et al. Neutrophil extracellular traps that are not degraded in systemic lupus erythematosus activate complement exacerbating the disease. J Immunol. 2012;188(7):3522-3531.

44. Lewis HD, et al. Inhibition of PAD4 activity is sufficient to disrupt mouse and human NET formation. Nat Chem Biol. 2015;11(3):189-191.

45. Kowalska MA, Rauova L, Poncz M. Role of the platelet chemokine platelet factor 4 (PF4) in hemostasis and thrombosis. Thromb Res. 2010;125(4):292-296.

46. Eslin DE, et al. Transgenic mice studies demonstrate a role for platelet factor 4 in thrombosis: dissociation between anticoagulant and antithrombotic effect of heparin. Blood. 2004;104(10):3173-3180.

47. Zhang C, et al. Localization of distal regulatory domains in the megakaryocyte-specific platelet basic protein/platelet factor 4 gene locus. Blood. 2001;98(3):610-617.

48. Cong L, Zhang F. Genome engineering using CRISPR-Cas9 system. Methods Mol Biol. 2015;1239:197-217.

49. Doench JG, et al. Optimized sgRNA design to maximize activity and minimize off-target effects of CRISPR-Cas9. Nat Biotechnol. 2016;34(2):184-191.

50. Cuker A, et al. The HIT Expert Probability (HEP) Score: a novel pre-test probability model for heparin-induced thrombocytopenia based on broad expert opinion. J Thromb Haemost. 2010;8(12):2642-2650.

51. Cuker A. Clinical and laboratory diagnosis of heparin-induced thrombocytopenia: an integrated approach. Semin Thromb Hemost. 2014;40(1):106-114

52. Arepally G, et al. Comparison of PF4/heparin ELISA assay with the 14C-serotonin release assay in the diagnosis of heparin-induced thrombocytopenia. Am J Clin Pathol. 1995;104(6):648-654.

53. Lambert MP, Gernsheimer TB. Clinical updates in adult immune thrombocytopenia. Blood. 2017;129(21):2829-2835.

54. Caudrillier A, et al. Platelets induce neutrophil extracellular traps in transfusion-related acute lung injury. J Clin Invest. 2012;122(7):2661-2671.

55. Tutwiler V, et al. Platelet transactivation by monocytes promotes thrombosis in heparin-induced thrombocytopenia. Blood. 2016;127(4):464-472.

56. Sachais BS, et al. Dynamic antibody-binding properties in the pathogenesis of HIT. Blood. 2012;120(5):1137-1142.

57. Drexler GA, Ruiz-Gómez MJ. Microirradiation techniques in radiobiological research. J Biosci. 2015;40(3):629-643

58. Schindelin J, et al. Fiji: an open-source platform for biological-image analysis. Nat Methods. 2012;9(7):676-682.

59. Neyman M, Gewirtz J, Poncz M. Analysis of the spatial and temporal characteristics of platelet-delivered factor VIII-based clots. Blood. 2008;112(4):1101-1108.

60. Holz O, et al. SCH527123, a novel CXCR2 antagonist, inhibits ozone-induced neutrophilia in healthy subjects. Eur Respir J. 2010;35(3):564-570 